\title{
ARTICLE
}

Genetics and Genomics

\section{NF1 mutations in conjunctival melanoma}

\author{
S. L. Scholz ${ }^{1}$, I. Cosgarea ${ }^{2}$, D. Süßkind ${ }^{3}$, R. Murali ${ }^{4}$, I. Möller ${ }^{2}$, H. Reis ${ }^{5}$, S. Leonardelli ${ }^{2}$, B. Schilling ${ }^{6}$, T. Schimming ${ }^{2}$, E. Hadaschik ${ }^{2}$, \\ C. Franklin ${ }^{2}$, A. Paschen ${ }^{2}$, A. Sucker ${ }^{2}$, K. P. Steuhl ${ }^{1}$, D. Schadendorf ${ }^{2}$, H. Westekemper ${ }^{1}$ and K. G. Griewank ${ }^{2,7}$
}

BACKGROUND: Conjunctival melanoma is a potentially deadly eye tumour. Despite effective local therapies, tumour recurrence and metastasis remain frequent. The genetics of conjunctival melanomas remain incompletely understood.

METHODS: A large cohort of 63 conjunctival melanomas was screened for gene mutations known to be important in other melanoma subtypes by targeted next-generation sequencing. Mutation status was correlated with patient prognosis.

RESULTS: Frequent mutations in genes activating the MAP kinase pathway were identified. NF1 mutations were most frequent $(n=21,33 \%)$. Recurrent activating mutations were also identified in BRAF ( $n=16,25 \%)$ and $R A S$ genes $(n=12,19 \% ; 11$ NRAS and 1 KRAS).

CONCLUSIONS: Similar to cutaneous melanomas, conjunctival melanomas can be grouped genetically into four groups:

BRAF-mutated, RAS-mutated, NF1-mutated and triple wild-type melanomas. This genetic classification may be useful for assessment of therapeutic options for patients with metastatic conjunctival melanoma

British Journal of Cancer (2018) 118:1243-1247; https://doi.org/10.1038/s41416-018-0046-5

\section{INTRODUCTION}

Conjunctival melanoma accounts for $5-10 \%$ of all ocular melanomas with a 10-year local recurrence rate of $38-69 \%$ and disease-related mortality of $13-38 \% .^{1-6} \mathrm{~A}$ better understanding of the genetics of conjunctival melanoma may help identify improved therapeutic options for patients with advanced disease.

In recent years, major melanoma subtypes have been genetically characterised. Cutaneous melanomas frequently harbour activating mutations in BRAF $(\sim 50 \%)^{7}$ or NRAS ( 20\%), as well as mutations in NF1..$^{8-11}$ BRAF, NRAS and NF1 mutations lead to activation of the mitogen-activated protein (MAP) kinase pathway. ${ }^{9}, 12,13$ Based on these findings, a genetic classification of cutaneous melanomas has been proposed distinguishing four genetic groups: BRAF-mutated, RAS-mutated, NF1-mutated or triple wild type. ${ }^{11}$

Uveal melanomas exhibit a different mutation profile, and harbour mutations in GNAQ ${ }_{1}^{14}$ GNA11, ${ }^{15}$ CYSLTR2, ${ }^{16}$ PLCB4, ${ }^{17}$ $E I F 1 A X^{18} S F 3 B 1^{19}$ and $B A P 1,{ }^{20}$ which are rarely found in other melanomas. $^{15,21-23}$

Conjunctival melanomas have not been characterised genetically as well as other melanoma subtypes. BRAF ${ }^{V 600 E}$ and NRAS mutations are present in $14-50 \%{ }^{24-27}$ and $18 \%{ }^{28}$ respectively, of conjunctival melanomas. TERT promoter mutations were identified in $32-41 \%$ of conjunctival melanomas. ${ }^{29,} 30$ One study reported a KIT mutation in $1 / 14$ (7\%) tumours. ${ }^{31}$ Copy number analysis identified alterations reminiscent of cutaneous and mucosal melanomas, including CDKN2A and PTEN losses. ${ }^{28}$ These data suggest that conjunctival melanomas are genetically similar to cutaneous melanomas, but aside from BRAF, NRAS and TERT promoter mutations, recurrent mutations in other genes have not been identified.

There are two main therapeutic avenues for metastatic melanoma. Firstly, targeted small inhibitors dampening pathologically activated cell-intrinsic signalling mechanisms, with the most effective to date being a combination of BRAF and MEK inhibitors in BRAF-mutated melanoma. ${ }^{32}$ Secondly, immunotherapies applying anti-CTLA-4 and anti-PD-1 antibodies have shown impressive response rates in cutaneous and mucosal melanoma. ${ }^{33-35}$ Both approaches may be clinically useful in advanced conjunctival melanoma. ${ }^{36}$

Our study aimed to further elucidate genetic events in conjunctival melanoma by analysing a large tumour cohort with a targeted next-generation sequencing assay covering genes that are recurrently mutated in cutaneous and uveal melanoma.

\section{MATERIALS AND METHODS}

Sample selection and histopathology

Sixty-seven conjunctival melanoma samples were obtained from the tissue archives of the Departments of Ophthalmology,

\footnotetext{
${ }^{1}$ Department of Ophthalmology, University Hospital Essen, Hufelandstrasse 55, Essen 45147, Germany; ${ }^{2}$ Department of Dermatology, University Hospital Essen, Hufelandstrasse 55, Essen 45147, Germany; ${ }^{3}$ Department of Ophthalmology, University Hospital Tübingen, Elfriede-Aulhorn-Strasse 7, Tübingen 72076, Germany; ${ }^{4}$ Department of Pathology, Memorial Sloan Kettering Cancer Center, 1275 York Avenue, New York, NY 10065, USA; ${ }^{5}$ Institute of Pathology, University Hospital Essen, West German Cancer Center, University Duisburg-Essen and the German Cancer Consortium (DKTK), Hufelandstrasse 55, Essen 45147, Germany; ${ }^{6}$ Department of Dermatology, University Hospital Würzburg, JosefSchneider-Straße 2, Würzburg 97080, Germany and ${ }^{7}$ Dermatopathologie bei Mainz, Bahnhofstr. 2b, Nieder-Olm 55268, Germany

Correspondence: S L. Scholz (simone.scholz@uk-essen.de) or K G. Griewank (klaus.griewank@uk-essen.de)

These authors contributed equally: H. Westekemper, K. G. Griewank.

These authors contributed equally: K. G. Griewank, S. L. Scholz.
}

Received: 3 December 2017 Revised: 27 January 2018 Accepted: 5 February 2018

Published online: 21 March 2018 
Table 1. Correlation between mutation status and clinical features in conjunctival melanomas

\begin{tabular}{|c|c|c|c|c|c|c|c|c|c|c|c|c|c|c|c|c|c|c|}
\hline & & \multicolumn{2}{|l|}{ Total } & \multicolumn{2}{|c|}{$B R A F^{W T}$} & \multicolumn{2}{|c|}{$B R A F^{V 600 E}$} & \multirow{2}{*}{$\begin{array}{l}P \text {-value } \\
(p<0.05)\end{array}$} & \multicolumn{2}{|c|}{$R A S^{W T}$} & \multicolumn{2}{|c|}{$R A S^{M U T}$} & \multirow{2}{*}{$\begin{array}{l}P \text {-value } \\
(p<0.05)\end{array}$} & \multicolumn{2}{|c|}{$N F 1^{W T}$} & \multicolumn{2}{|c|}{$N F 1^{M U T}$} & \multirow{2}{*}{$\begin{array}{l}P \text {-value } \\
(p<0.05)\end{array}$} \\
\hline & & $\%$ & $(n)$ & $\%$ & $(n)$ & $\%$ & $(n)$ & & $\%$ & $(n)$ & $\%$ & $(n)$ & & $\%$ & $(n)$ & $\%$ & $(n)$ & \\
\hline Total & & & 63 & 74.6 & 47 & 25.4 & 16 & & 81.0 & 51 & 19.0 & 12 & & 66.7 & 42 & 33.3 & 21 & \\
\hline \multirow[t]{2}{*}{ Sex } & Female & 50.8 & 32 & 38.1 & 24 & 12.7 & 8 & 0.94 & 44.4 & 28 & 6.3 & 4 & 0.18 & 36.5 & 23 & 14.3 & 9 & 0.37 \\
\hline & Male & 49.2 & 31 & 36.5 & 23 & 12.7 & 8 & & 36.5 & 23 & 12.7 & 8 & & 30.2 & 19 & 19 & 12 & \\
\hline \multirow[t]{3}{*}{ Eye } & Right & 55.6 & 35 & 39.7 & 25 & 15.9 & 10 & 0.63 & 42.9 & 27 & 12.7 & 8 & 0.59 & 34.9 & 22 & 20.6 & 13 & 0.52 \\
\hline & Left & 41.3 & 26 & 31.7 & 20 & 9.5 & 6 & & 34.9 & 22 & 6.3 & 4 & & 28.6 & 18 & 12.7 & 8 & \\
\hline & $\mathrm{N} / \mathrm{A}$ & 3.2 & 2 & 3.2 & 2 & 0 & 0 & & 3.2 & 2 & 0 & 0 & & 3.2 & 2 & 0 & 0 & \\
\hline \multirow[t]{4}{*}{ TNM } & 1 & 55.6 & 35 & 41.3 & 26 & 14.3 & 9 & 0.77 & 44.4 & 28 & 11.1 & 7 & 0.44 & 38.1 & 24 & 17.5 & 11 & 0.56 \\
\hline & 2 & 23.8 & 15 & 17.5 & 11 & 6.3 & 43 & & 22.2 & 14 & 1.6 & 1 & & 14.3 & 9 & 9.5 & 6 & \\
\hline & 3 & 15.9 & 10 & 11.1 & 7 & 4.8 & 0 & & 11.1 & 7 & 4.8 & 3 & & 9.5 & 6 & 6.3 & 4 & \\
\hline & N/A & 4.8 & 3 & 4.8 & 3 & 0 & & & 3.2 & 2 & 1.6 & 1 & & 4.8 & 3 & 0 & 0 & \\
\hline \multirow[t]{4}{*}{ Tumour origin } & PAM & 52.4 & 33 & 41.3 & 26 & 11.1 & 7 & 0.1 & 44.4 & 28 & 7.9 & 5 & 0.35 & 37.5 & 24 & 14.3 & 9 & 0.43 \\
\hline & Naevus & 17.5 & 11 & 7.9 & 5 & 9.5 & 6 & & 15.9 & 10 & 1.6 & 1 & & 11.1 & 7 & 6.3 & 4 & \\
\hline & De novo & 22.2 & 14 & 19 & 12 & 3.2 & 2 & & 15.9 & 10 & 4.8 & 4 & & 11.1 & 7 & 11.1 & 7 & \\
\hline & $\mathrm{N} / \mathrm{A}$ & 7.9 & 5 & 6.3 & 4 & 1.6 & 1 & & 4.8 & 3 & 3.2 & 2 & & 6.3 & 4 & 1.6 & 1 & \\
\hline \multirow[t]{3}{*}{ Relapses } & No & 46 & 29 & 36.5 & 23 & 9.5 & 6 & 0.26 & 33.3 & 21 & 12.7 & 8 & 0.22 & 27 & 17 & 19 & 12 & 0.46 \\
\hline & Yes & 47.6 & 30 & 31.7 & 20 & 15.9 & 10 & & 42.9 & 27 & 4.8 & 3 & & 34.9 & 22 & 12.7 & 8 & \\
\hline & $\mathrm{N} / \mathrm{A}$ & 6.3 & 4 & 6.3 & 4 & 0 & 0 & & 4.8 & 3 & 1.6 & 1 & & 4.8 & 3 & 1.6 & 1 & \\
\hline \multirow[t]{3}{*}{ Metastasis } & No & 68.3 & 43 & 49.2 & 31 & 19.0 & 12 & 0.32 & 54.0 & 34 & 14.3 & 9 & 0.34 & 42.9 & 27 & 25.4 & 16 & 0.55 \\
\hline & Yes & 22.2 & 14 & 15.9 & 10 & 6.3 & 4 & & 20.6 & 13 & 1.6 & 1 & & 17.5 & 11 & 4.8 & 3 & \\
\hline & $\mathrm{N} / \mathrm{A}$ & 9.5 & 6 & 9.5 & 6 & 0 & 0 & & 6.3 & 4 & 3.2 & 2 & & 6.3 & 4 & 3.2 & 2 & \\
\hline \multirow[t]{3}{*}{ Exenteration } & No & 76.2 & 48 & 57.1 & 36 & 19.0 & 12 & 0.36 & 60.3 & 38 & 15.9 & 10 & 0.64 & 47.6 & 30 & 28.6 & 18 & 0.44 \\
\hline & Yes & 17.5 & 11 & 11.1 & 7 & 6.3 & 4 & & 15.9 & 10 & 1.6 & 1 & & 14.3 & 9 & 3.2 & 2 & \\
\hline & $\mathrm{N} / \mathrm{A}$ & 6.3 & 4 & 6.3 & 4 & 0 & 0 & & 4.8 & 3 & 1.6 & 1 & & 4.8 & 3 & 1.6 & 1 & \\
\hline Age at diagnose & \multicolumn{18}{|c|}{ Median 67.4 years, Range $40.1-88.8$ years } \\
\hline
\end{tabular}

Dermatology and Pathology of the University Hospital Essen, and the Department of Ophthalmology, University Hospital Tübingen, Germany. The study was approved by the local ethics committee of the University of Duisburg-Essen.

DNA isolation

Formalin-fixed, paraffin-embedded tumour tissues were sectioned, deparaffinised and manually microdissected as previously described. ${ }^{37}$ Genomic DNA was isolated using the QIAamp DNA Mini Kit (Qiagen, Hilden, Germany).

\section{Targeted sequencing}

A custom amplicon-based sequencing panel covering 29 genes known to be mutated in melanoma was used (genes listed in Supplemental Table 1), as previously described. ${ }^{37}$ Mean coverage of 2094 reads, with a minimum coverage of 30 reads in $>80 \%$ of the target loci, was achieved. Four samples were excluded from analysis due to low coverage.

Sequence analysis

CLC Cancer Research Workbench from QIAGEN ${ }^{\circledR}$ was used for sequence analysis, as previously reported. ${ }^{37}$ Mutations were considered if coverage of the mutation site was $\geq 30$ reads, $\geq 10$ reads reported the mutated variant and the frequency of mutated reads was $\geq 10 \%$.
Associations of mutation status with clinical and pathological parameters

Associations of mutation status with available clinicopathological parameters (listed in Table 1) were explored. Analyses were performed with IBM SPSS Statistics software (version 20.0; International Business Machines Corp., Armonk NY, USA). A $p$-value of $<0.05$ was considered statistically significant.

\section{RESULTS}

Tumours and patients

Conjunctival melanomas occurred equally in male and female with a median age of 67 years (range 40-89 years). Of the samples for which information was available, $52 \%$ $(33 / 63)$ originated from primary acquired melanosis (PAM), 18\% $(11 / 63)$ from naevi and $22 \%$ (14/63) arose de novo. Clinical stage at initial presentation was stage 1 , stage 2 and stage 3 in $56 \%(35 / 63), 24 \%(15 / 63)$ and $16 \%(10 / 63)$ of patients, respectively (American Joint Committee on Cancer staging system for conjunctival melanoma, 7th edition, 2010). Adjuvant treatment was received by $87 \%(55 / 63)$ of patients (21 ruthenium, 17 proton, 6 percutaneous radiotherapy, 7 cryotherapy and 3 mitomycin C). Tumours recurred in $47 \%(30 / 63)$ and metastasised in $22 \%(14 / 63)$ of cases. Additional information is listed in Table 1. 


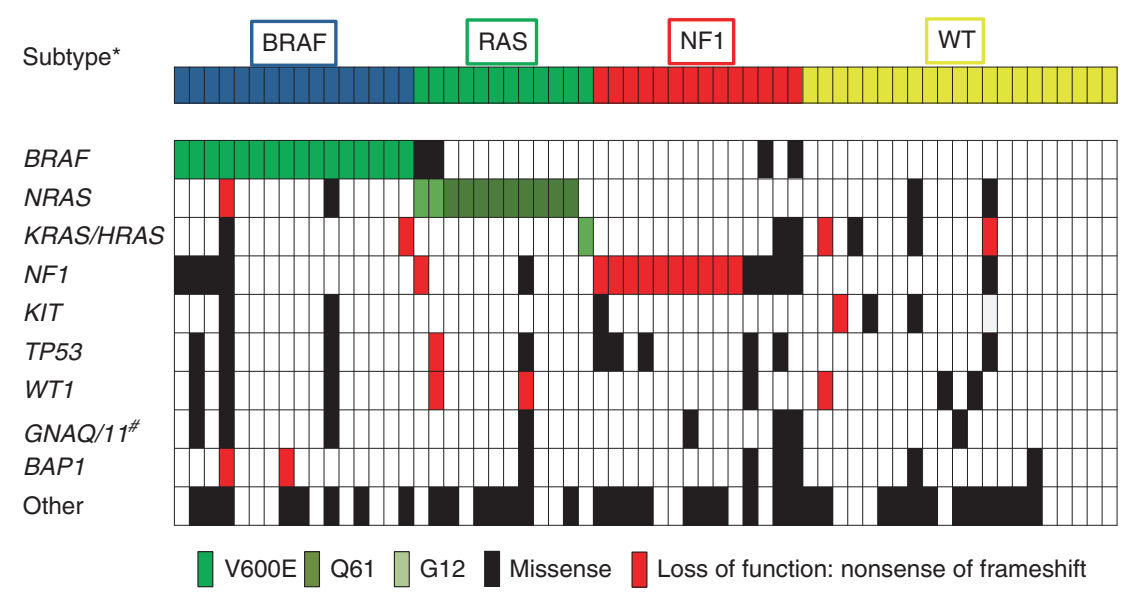

Fig. 1 Mutations in conjunctival melanoma. Distribution of mutations identified by amplicon panel next-generation sequencing. Green: mutations known or assumed to be activating; Red: nonsense or frameshift loss-of-function mutations; Black: missense mutation with unknown functional consequences. Mutations listed as "Other" include mutations detected in CDK4, FLT4, PIK3CA, PIK3R1, FBXW7, MITF, MAP2K1, MAP2K2, ARID1A, ARID2, SF3B1, CTNNB1, PTEN, CDKN2A, SMARCA4A, EZH2, IDH1 and the protein-coding area of TERT (the promoter region of TERT was not covered by the amplicon-based sequencing panel used in this study). *Subtype according to TCGA genomic classification of cutaneous melanoma. "None of the GNAQ or GNA11 mutations identified were the known activating Q209 or R183 mutations recurrently identified in uveal melanomas (details in Supplemental Table 2)

Identified mutations

Activating $B R A F^{V 600 E}$ (c.1799A $>\mathrm{T}$ ) mutations were detected in 16/63 (25\%) tumours. Additionally, 4 BRAF mutations with unknown functional consequences were identified (Supplemental Table 2).

Activating RAS mutations (11 NRAS and 1 KRAS mutation) were identified in $12 / 63$ (19\%) tumours (Table 2). We also detected 4 NRAS, 3 KRAS and 5 HRAS mutations with unknown functional consequences (Supplemental Table 2).

NF1 mutations were identified in 21/63 (33\%) tumours. Clearly inactivating NF1 mutations were observed in 10 tumours. NF1 mutations co-occurred with BRAF and RAS gene mutations in some tumours, but also frequently occurred alone. All identified mutations are shown in Fig. 1 and listed in Table 2, and shown in Supplemental Figs. 1 and 2.

Additionally, mutations in various genes frequently mutated in cutaneous melanoma were detected. The majority of these mutations were of unknown functional consequences (Supplemental Table 2). While a few GNAQ and GNA11 mutations were identified (Fig. 1, Supplemental Table 2), they presumably represent functionally non-relevant bystander mutations, as none of the identified mutations were the activating R183 or Q209 mutations known to occur in uveal melanomas. $^{14,15,21}$

Statistical analysis

There were no statistically significant associations between clinicopathological parameters with $B R A F, R A S$ and NF1 mutation status (Table 1).

\section{DISCUSSION}

To our knowledge, the present study represents the most detailed analysis of gene mutations in conjunctival melanoma to date.

Activating BRAF mutations were detected in $25 \%$ of samples, lying within the range of previous studies reporting $14-50 \% .^{24-27,} 38$ This variation may be due to sample bias or technical differences. In view of the recent development of effective BRAF and MEK inhibitors, the presence of BRAF V600 mutations in conjunctival melanomas is of considerable therapeutic relevance. ${ }^{39}$

In addition to known activating NRAS mutations in $18 \%$ $(11 / 63)$ of tumours, we identified an activating KRAS G12A mutation. Being the first report on these mutations in conjunctival melanoma, this finding is reminiscent of cutaneous melanoma, in which KRAS mutations are rare but occur in a mutually exclusive fashion with NRAS mutations. ${ }^{11}$ In the proposed TCGA (The Cancer Genome Atlas) genomic classification of cutaneous melanoma, mutations in all three RAS genes are grouped together as RAS-mutated melanomas.

Our study is the first to identify $N F 1$ as a frequently mutated oncogene (33\%) in conjunctival melanoma. NF1 has recently been recognised as the third most commonly mutated gene (after BRAF and RAS) in cutaneous melanoma, activating the MAP kinase pathway. ${ }^{11}$ In our conjunctival melanoma cohort, NF1 mutations were also present in samples harbouring activating RAS or BRAF mutations (Fig. 1). This is similar to the situation in cutaneous melanoma where the co-occurrence of NF1 with BRAF, RAS and other mutations is well recognised. ${ }^{11,12,40}$

NF1 mutations are particularly frequent in melanoma subtypes rarely harbouring BRAF and NRAS mutations, $8,12,41$ including melanomas associated with high sun exposure. ${ }^{8,} 12$ Ultraviolet exposure is a known pathogenic factor in conjunctival melanoma and could explain the high number of NF1 mutations detected. NF1 mutations have been associated with high tumour mutational load and affected patients have been reported to benefit from anti-PD-1 therapy in cutaneous melanoma. ${ }^{42}$ This suggests that NF1 mutation status has potential as a biomarker for immunotherapy in conjunctival melanoma.

In summary, our study identifies a range of mutations in conjunctival melanoma. The distribution of activating mutations, with RAS gene mutations occurring not only in NRAS but also KRAS, and NF1 mutations being frequent in tumours lacking BRAF or RAS mutations, suggests that the proposed genetic classification of cutaneous melanomas into BRAF-mutated, RAS-mutated, NF1-mutated or triple-wild-type tumours is also applicable to conjunctival melanoma. 
Table 2. MAP kinase pathway activating mutations in conjunctival melanoma

\begin{tabular}{|c|c|c|c|c|}
\hline \multirow[t]{2}{*}{ Gene } & & \multirow[t]{2}{*}{ Mutation type } & \multicolumn{2}{|c|}{$\begin{array}{l}\text { Tumours harbouring } \\
\text { mutation }\end{array}$} \\
\hline & & & $N$ & $\%$ \\
\hline \multirow{2}{*}{\multicolumn{2}{|c|}{ BRAF }} & All mutations & 16 & 25 \\
\hline & & V600E & 16 & 25 \\
\hline \multirow[t]{8}{*}{ RAS } & & All mutations & 12 & 19 \\
\hline & NRAS & Q61R & 5 & 8 \\
\hline & & Q61K & 2 & 3 \\
\hline & & Q61H & 1 & 2 \\
\hline & & Q61L & 1 & 2 \\
\hline & & G13D & 1 & 2 \\
\hline & & $\mathrm{G} 12 \mathrm{~N}, \mathrm{G} 12 \mathrm{C}$ & 1 & 2 \\
\hline & KRAS & G12A & 1 & 2 \\
\hline \multirow[t]{21}{*}{ NF1 } & & All mutations & 21 & 33 \\
\hline & & T60del & 1 & 2 \\
\hline & & $\mathrm{R} 262 \mathrm{C}$ & 1 & 2 \\
\hline & & C42Y, G2397R, S2587L & 1 & 2 \\
\hline & & S2751N, L552P, G2392E & 1 & 2 \\
\hline & & D176E & 2 & 3 \\
\hline & & L847P, P866S, V1762I & 1 & 2 \\
\hline & & C1899Y & 1 & 2 \\
\hline & & M1180I, S52F; T60l & 1 & 2 \\
\hline & & A2715V; A2208T & 1 & 2 \\
\hline & & G2397R, R2517fs & 1 & 2 \\
\hline & & I1824fs & 1 & 2 \\
\hline & & $\mathrm{L}^{1892^{\mathrm{a}}}$ & 1 & 2 \\
\hline & & N1451L & 1 & 2 \\
\hline & & Q1815 & 1 & 2 \\
\hline & & Q756fs & 1 & 2 \\
\hline & & $\mathrm{R} 1362^{\mathrm{a}}$ & 1 & 2 \\
\hline & & $\begin{array}{l}\text { R440, }{ }^{\mathrm{a}}, \mathrm{Q} 2239^{\mathrm{a}} ; \mathrm{S} 1497 \mathrm{~F} ; \\
\text { V1393A }\end{array}$ & 1 & 2 \\
\hline & & S168L & 1 & 2 \\
\hline & & $\mathrm{S} 1786^{\mathrm{a}}, \mathrm{L} 1102^{\mathrm{a}} ; \mathrm{Q} 1815 \mathrm{fs}$ & 1 & 2 \\
\hline & & Y1678fs & 1 & 2 \\
\hline $\begin{array}{l}\text { Wild } \\
\text { type }\end{array}$ & & & 14 & 22 \\
\hline Total & & & 63 & \\
\hline
\end{tabular}

MAP mitogen-activated protein, fs frameshift mutations ${ }^{\mathrm{a}}$ Nonsense mutations

\section{ACKNOWLEDGEMENTS}

We would like to thank Nadine Stadler and Nicola Bielefeld for their excellent technical support. The research was supported by a grant from Dr. Werner-JackstädtStiftung (www.jackstaedt-stiftung.de) and Hiege-Stiftung gegen Hautkrebs. This work was also funded in part through the $\mathrm{NIH} / \mathrm{NCl}$ Cancer Center Support Grant P30 CA008748 and received funding from the European Union's Horizon 2020 research and innovation programme under the Marie Sklodowska-Curie grant agreement No 641458. The funders had no role in study design, data collection and analysis, decision to publish or preparation of the manuscript.

\section{AUTHOR CONTRIBUTIONS}

Literature search: S.L.S., K.G.G., B.S., H.W., R.M.; study design: K.G.G., K.P.S., D.S.; data collection: S.L.S., I.C., D.S., R.M., I.M., H.R., S.T., S.L., E.H., C.F., K.G.G., H.W., A.P., D.S.; data analysis: S.L.S., K.G.G., R.M., T.S., H.W., A.S., A.P.; data interpretation: S.L.S., K.G.G., A.P., A.S., H.W.; manuscript writing: all authors.

\section{ADDITIONAL INFORMATION}

Supplementary information is available for this paper at https://doi.org/10.1038/ s41416-018-0046-5.

Competing interests: Dirk Schadendorf is on the advisory board or has received honararia from Roche, Genetech, Novartis, Amgen, GSK, Boehringer Ingelheim and Merck. The other authors declare no competing interests.

Note: This work is published under the standard license to publish agreement. After 12 months the work will become freely available and the license terms will switch to a Creative Commons Attribution 4.0 International licence (CC BY 4.0).

\section{REFERENCES}

1. Harooni, H., Schoenfield, L. R. \& Singh, A. D. Current appraisal of conjunctival melanocytic tumors: classification and treatment. Future Oncol. 7, 435-446 (2011).

2. Shields, C. L. et al. Conjunctival melanoma: risk factors for recurrence, exenteration, metastasis, and death in 150 consecutive patients. Arch. Ophthalmol. 118, 1497-1507 (2000)

3. Paridaens, A. D., McCartney, A. C., Minassian, D. C. \& Hungerford, J. L. Orbital exenteration in 95 cases of primary conjunctival malignant melanoma. Br. J. Ophthalmol. 78, 520-528 (1994).

4. Werschnik, C. \& Lommatzsch, P. K. Long-term follow-up of patients with conjunctival melanoma. Am. J. Clin. Oncol. 25, 248-255 (2002).

5. Missotten, G. S., Keijser, S., De Keizer, R. J. \& De Wolff-Rouendaal, D. Conjunctival melanoma in the Netherlands: a nationwide study. Invest. Ophthalmol. Vis. Sci. 46, 75-82 (2005).

6. Shildkrot, Y. \& Wilson, M. W. Conjunctival melanoma: pitfalls and dilemmas in management. Curr. Opin. Ophthalmol. 21, 380-386 (2010).

7. Davies, $\mathrm{H}$. et al.Mutations of the BRAF gene in human cancer. Nature 417, 949-954 (2002).

8. Wiesner, T. et al. NF1 mutations are common in desmoplastic melanoma. Am. J. Surg. Pathol. 39, 1357-1362 (2015).

9. Shain, A. H. et al. Exome sequencing of desmoplastic melanoma identifies recurrent NFKBIE promoter mutations and diverse activating mutations in the MAPK pathway. Nat. Genet. 47, 1194-1199 (2015).

10. Viskochil, D. et al. Deletions and a translocation interrupt a cloned gene at the neurofibromatosis type 1 locus. Cell 62, 187-192 (1990).

11. Cancer Genome Atlas Network. Genomic classification of cutaneous melanoma. Cell 161, 1681-1696 (2015).

12. Krauthammer, M. et al. Exome sequencing identifies recurrent mutations in NF1 and RASopathy genes in sun-exposed melanomas. Nat. Genet. 47, 996-1002 (2015).

13. Griewank, K. G. et al. Genetic alterations and personalized medicine in melanoma: progress and future prospects. J. Natl. Cancer Inst. 106, djt435 (2014).

14. Van Raamsdonk, C. D. et al. Frequent somatic mutations of GNAQ in uveal melanoma and blue naevi. Nature 457, 599-602 (2009).

15. Van Raamsdonk, C. D. et al. Mutations in GNA11 in uveal melanoma. N. Engl. J. Med. 363, 2191-2199 (2010).

16. Moore, A. R. et al. Recurrent activating mutations of G-protein-coupled receptor CYSLTR2 in uveal melanoma. Nat. Genet. 48, 675-680 (2016).

17. Johansson, P. et al. Deep sequencing of uveal melanoma identifies a recurrent mutation in PLCB4. Oncotarget 7, 4624-4631 (2016).

18. Martin, M. et al. Exome sequencing identifies recurrent somatic mutations in EIF1AX and SF3B1 in uveal melanoma with disomy 3. Nat. Genet 45, 933-936 (2013).

19. Harbour, J. W. et al. Recurrent mutations at codon 625 of the splicing factor SF3B1 in uveal melanoma. Nat. Genet. 45, 133-135 (2013).

20. Harbour, J. W. et al. Frequent mutation of BAP1 in metastasizing uveal melanomas. Science 330, 1410-1413 (2010).

21. Robertson, A. G. et al. Integrative analysis identifies four molecular and clinical subsets in uveal melanoma. Cancer Cell. 32, 204-220 (2017). e15.

22. Griewank, K. G. et al. SF3B1 and BAP1 mutations in blue nevus-like melanoma. Mod. Pathol. 30, 928-939 (2017).

23. van de Nes, J. A. P. et al. Activating CYSLTR2 and PLCB4 mutations in primary leptomeningeal melanocytic tumors. J. Invest. Dermatol. 137, 2033-2035 (2017).

24. Spendlove, H. E. et al. BRAF mutations are detectable in conjunctival but not uveal melanomas. Melanoma Res. 14, 449-452 (2004). 
25. Lake, S. L. et al. Multiplex ligation-dependent probe amplification of conjunctival melanoma reveals common BRAF V600E gene mutation and gene copy number changes. Invest. Ophthalmol. Vis. Sci. 52, 5598-5604 (2011).

26. Goldenberg-Cohen, N. et al. T1799A BRAF mutations in conjunctival melanocytic lesions. Invest. Ophthalmol. Vis. Sci. 46, 3027-3030 (2005).

27. Gear, H., Williams, H., Kemp, E. G. \& Roberts, F. BRAF mutations in conjunctival melanoma. Invest. Ophthalmol. Vis. Sci. 45, 2484-2488 (2004).

28. Griewank, K. et al. Conjunctival melanomas harbor BRAF and NRAS mutations and copy number changes similar to cutaneous and mucosal melanomas. Clin. Cancer Res. 19, 3143-3152 (2013).

29. Griewank, K. G. et al. TERT promoter mutations in ocular melanoma distinguish between conjunctival and uveal tumours. Br. J. Cancer 109, 497-501 (2013).

30. Koopmans, A. E. et al. Prevalence and implications of TERT promoter mutation in uveal and conjunctival melanoma and in benign and premalignant conjunctival melanocytic lesions. Invest. Ophthalmol. Vis. Sci. 55, 6024-6030 (2014).

31. Beadling, C. et al. KIT gene mutations and copy number in melanoma subtypes. Clin. Cancer Res. 14, 6821-6828 (2008).

32. Robert, C. et al. Improved overall survival in melanoma with combined dabrafenib and trametinib. N. Engl. J. Med. 372, 30-39 (2015).

33. Robert, $C$. et al. Pembrolizumab versus Ipilimumab in Advanced Melanoma. $N$. Engl. J. Med. 372, 2521-2532 (2015).
34. Larkin, J. et al. Combined nivolumab and ipilimumab or monotherapy in untreated melanoma. N. Engl. J. Med. 373, 23-34 (2015).

35. Schadendorf, D. et al. Pooled analysis of long-term survival data from phase II and phase III trials of ipilimumab in unresectable or metastatic melanoma. J. Clin. Oncol. 33, 1889-1894 (2015).

36. Griewank, K. G. et al. Conjunctival melanomas harbor BRAF and NRAS mutations-response. Clin. Cancer Res. 19, 6331-6332 (2013).

37. van de Nes, J. et al. Targeted next generation sequencing reveals unique mutation profile of primary melanocytic tumors of the central nervous system. $J$. Neuro oncol. 127, 435-444 (2016).

38. Larsen, A. C. et al. BRAF mutations in conjunctival melanoma: investigation of incidence, clinicopathological features, prognosis and paired premalignant lesions. Acta Ophthalmol. 94, 463-470 (2016).

39. Cao, J. et al. Targeting of the MAPK and AKT pathways in conjunctival melanoma shows potential synergy. Oncotarget 8, 58021-58036 (2017).

40. Nissan, M. H. et al. Loss of NF1 in cutaneous melanoma is associated with RAS activation and MEK dependence. Cancer Res. 74, 2340-2350 (2014).

41. Cosgarea, I. et al. Targeted next generation sequencing of mucosal melanomas identifies frequent NF1 and RAS mutations. Oncotarget 8, 40683-40692 (2017).

42. Johnson, D. B. et al. Targeted next generation sequencing identifies markers of response to PD-1 blockade. Cancer Immunol. Res. 4, 959-967 (2016). 